STRUCTURAL VIROLOGY

\title{
Structural basis of synergistic neutralization of Crimean-Congo hemorrhagic fever virus by human antibodies
}

\author{
Akaash K. Mishra ${ }^{1} \dagger$, Jan Hellert ${ }^{2} \dagger \ddagger$, Natalia Freitas ${ }^{3}$, Pablo Guardado-Calvo ${ }^{2}$, Ahmed Haouz ${ }^{4}$, \\ J. Maximilian Fels ${ }^{5} \S$, Daniel P. Maurer ${ }^{6}$, Dafna M. Abelson ${ }^{7}$, Zachary A. Bornholdt', Laura M. Walker ${ }^{6}$, \\ Kartik Chandran ${ }^{5}$, François-Loïc Cosset ${ }^{3}$, Jason S. McLellan ${ }^{1 *}$, Felix A. Rey ${ }^{2 *}$
}

\begin{abstract}
Crimean-Congo hemorrhagic fever virus (CCHFV) is the most widespread tick-borne zoonotic virus, with a $30 \%$ case fatality rate in humans. Structural information is lacking in regard to the CCHFV membrane fusion glycoprotein Gc-the main target of the host neutralizing antibody response-as well as antibody-mediated neutralization mechanisms. We describe the structure of prefusion Gc bound to the antigen-binding fragments (Fabs) of two neutralizing antibodies that display synergy when combined, as well as the structure of trimeric, postfusion Gc. The structures show the two Fabs acting in concert to block membrane fusion, with one targeting the fusion loops and the other blocking Gc trimer formation. The structures also revealed the neutralization mechanism of previously reported antibodies against CCHFV, providing the molecular underpinnings essential for developing CCHFVspecific medical countermeasures for epidemic preparedness.
\end{abstract}

〔 rimean-Congo hemorrhagic fever virus (CCHFV) is endemic to Africa, Asia, and Europe and is transmitted by ticks and contact with bodily fluids from viremic animals or patients $(1,2)$. Although infection is asymptomatic in most vertebrates, it can cause severe disease in humans, with hemorrhage, myalgia, and high fever, eventually leading to death in $\sim 30 \%$ of diagnosed cases $(1,3,4)$. As a result, the World Health Organization has shortlisted CCHFV as a priority pathogen in its research and development blueprint (5). The Balkan peninsula and Turkey bear the highest burden; however, global warming facilitates the spread of the tick vector into new habitats through transport by migratory birds, as exemplified by a recent outbreak in Spain and the appearance of infected ticks in Italy $(6-8)$.

CCHFV is a member of the Orthonairovirus genus in the Nairoviridae family of the Bunyavirales order of viruses with a segmented,

\footnotetext{
Department of Molecular Biosciences, The University of Texas at Austin, Austin, TX 78712, USA. 'Institut Pasteur, Université de Paris, CNRS UMR 3569, Structural Virology Unit, 25-28 rue du Docteur Roux, Cedex 15, Paris, 75724 France. ${ }^{3} \mathrm{CIRI}$-Centre International de Recherche en Infectiologie, Univ Lyon, Université Claude Bernard Lyon 1, Inserm, U1111, CNRS, UMR5308, ENS Lyon, 46 allée d'Italie, Lyon, 69007 France. ${ }^{4}$ Institut Pasteur, Université de Paris, CNRS UMR 3528, Crystallography Platform C2RT, 25-28 rue du Docteur Roux, Cedex 15, Paris, 75724 France. ${ }^{5}$ Department of Microbiology and Immunology, Albert Einstein College of Medicine, Bronx, NY 10461, USA. ${ }^{6}$ Adimab LLC, Lebanon, NH 03766, USA. ${ }^{7}$ Mapp Biopharmaceutical Inc., San Diego, CA 92121, USA.

*Corresponding author. Email: jmclellan@austin.utexas.edu (J.S.M.); felix.rey@pasteur.fr (F.A.R)

†These authors contributed equally to this work. łPresent address: Centre for Structural Systems Biology, Leibniz-Institut für Experimentelle Virologie (HPI), Notkestraße 85, 22607 Hamburg, Germany. §Present address: Departments of Cell Biology and Microbiology, Harvard Medical School, Boston, MA, USA. ๆPresent address: Department of Cancer Immunology and Virology, DanaFarber Cancer Institute, Boston, MA, USA.
}

negative-strand RNA genome (9). New human pathogens in the Orthonairovirus genus (termed nairoviruses from here on) continue to be identified (10), highlighting the need for highresolution structural information to guide antiviral strategies. The Bunyavirales order also includes other pathogenic arthropod-borne viruses ("arboviruses") such as the Rift Valley fever virus (RVFV, Phlebovirus genus, Phenuiviridae family) and rodent-borne viruses such as Andes virus (Orthohantavirus genus, Hantaviridae family). CCHFV infects host cells through its envelope glycoproteins Gn and Gc, which form a locally ordered lattice of heterodimers on the virus surface after they are cleaved from a poly-glycoprotein precursor by host proteases (Fig. 1A) (11-13). Entry into target cells takes place by receptor-mediated endocytosis (14), with the acidic environment of the endosome triggering dissociation of the Gn-Gc heterodimer and the surface lattice, followed by a conformational change of Gc into a trimer of hairpin structures to drive membrane fusion (Fig. 1B). As with most bunyaviruses, CCHFV Gc is predicted to be a class II membrane fusion protein $(11,12)$ and is the only known target of CCHFV-neutralizing antibodies (15).

We determined the $\mathrm{x}$-ray structure of the CCHFV Gc postfusion trimer using two constructs at resolutions of 2.2 and $3.0 \AA$ (table S1), as described in the materials and methods. The trimer revealed a typical class II fold, with each protomer adopting the characteristic postfusion hairpin conformation (16). The inner arm of this hairpin is composed of domains I and II (red and yellow, respectively; Fig. 1C) and forms a rodlike structure with the distal tip of domain II exposing loops $b c, c d$, and $i j$, also termed "fusion loops" as they form a nonpolar host-membrane insertion surface (HMIS) required to drive membrane fusion. The domain I and II rods interact about the threefold molecular axis along their entire length to make an elongated trimeric core. The outer arm of the hairpin is formed by domain III (blue) followed by the stem (magenta) running in an extended conformation to reach the HMIS, thus completing the hairpin by bringing the downstream C-terminal transmembrane segment (not included in our structure) next to the HMIS. The turn of the hairpin at the opposite end of the rod is made of a linker region connecting domains I and III (Fig. 1C, cyan). Domain III and the stem together fill the cleft between two neighboring subunits of the core trimer, contributing to the stability of the postfusion conformation of Gc. The overall arrangement of domains I and III is similar to that of the fusion proteins of other arboviruses such as phleboviruses $(17,18)$, flaviviruses $(19,20)$, and alphaviruses (21). This organization is different, however, in hantaviruses $(22,23)$ and rubella virus (24), which do not infect arthropods. In the class II fusion proteins of these mammal-specific viruses, domain III is exchanged between neighboring protomers in the trimer (fig. S1).

Among the most potently neutralizing human monoclonal antibodies (mAbs) targeting CCHFV Gc, ADI-36121 and ADI-37801 were synergistic in coneutralization experiments (15). We determined the x-ray structure of both antigen-binding fragments (Fabs) in a ternary complex with monomeric Gc to $2.1-\AA$ resolution (table $\mathrm{S} 1$ ) as described in the materials and methods. The structure showed a Gc monomer with the ADI-36121 Fab bound at the domain II base and the ADI-37801 Fab bound at the HMIS (Fig. 1D). The crystals provided interpretable electron density only for domains I and II, as well as part of the linker between domains I and III, indicating that the whole outer arm observed in the postfusion hairpin is mobile in the monomer. On the virion surface, however, the prefusion conformation of Gc is likely further stabilized by contact with Gn and neighboring Gn-Gc heterodimers. Compared with the Gc postfusion trimer, the conformation of domain I in the monomer is different. In particular, the $\mathrm{N}$ - and C-terminal $\beta$ strands, $\mathrm{A}_{0}$ and $\mathrm{J}_{0}$, display an altered topology (fig. S1A). A similar change in the conformation of domain I has been observed between the pre- and postfusion structures of phlebovirus Gc $(17,18,25)$ (fig. S1B), indicating that the conformation of domain I in the CCHFV Gc monomer indeed corresponds to the prefusion form.

Unlike domain I, the conformation of the domain II tip in the Fab-bound monomer is similar to that seen in the postfusion trimer (Fig. 2A). In the flavivirus, alphavirus, and phlebovirus class II fusion proteins, the HMIS 


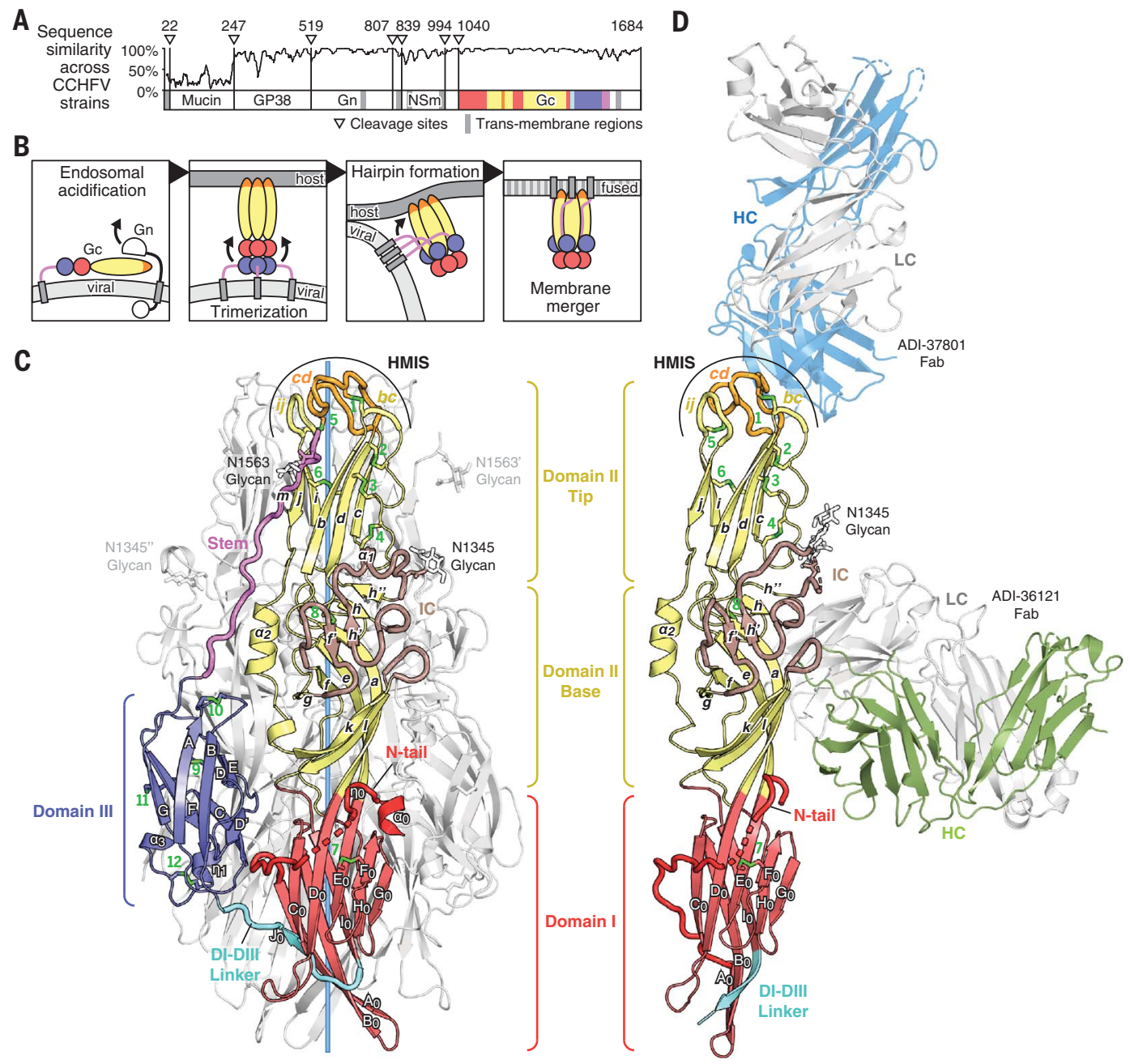

Fig. 1. Structures of CCHFV

Gc. (A) Organization of the

CCHFV glycoprotein precursor

(B) Mechanism of bunyavirus

class II membrane fusion

proteins. (C) X-ray structure of the CCHFV Gc ectodomain in the postfusion conformation. The front protomer is colored according to domain, and the trimer axis is shown in light blue. Secondary structure elements and disulfide bonds (green numbers) are labeled. An orthonairovirus-specific insertions cluster (IC) is depicted in brown. (D) X-ray structure of the CCHFV Gc monomer in complex with the ADI-37801 and ADI-36121 Fabs. HC, heavy chain; LC, light chain. is formed exclusively by the $c d$ loop (orange in our figures) (16). In hantaviruses, however, the HMIS is tripartite, with additional contributions from two adjacent loops, $b c$ and $i j$ (Fig. 2B) (22). CCHFV Gc has a similar tripartite configuration at its domain II tip, sharing a pattern of conserved residues with hantavirus Gc (Fig. 2C) despite an overall sequence identity of only $\sim 20 \%$ between the two Gc orthologs. Fig. 2 compares the CCHFV Gc to that of Maporal virus (MPRLV), for which best-resolved pre- and postfusion hantavirus Gc structures are available $(22,23,26)$. The main-chain conformation of the $b c, c d$, and $i j$ loops is similar in the postfusion forms of the CCHFV and MPRLV Gc (Fig. 2, A and B, left panels), with a root-mean-square deviation (RMSD) of $0.8 \AA \AA$ over $29 \mathrm{C} \alpha$ atom pairs. In both cases, four conserved disulfide bonds (Fig. 2, A to C, green) stabilize the structure, two of which cross-link the $c d$ loop with the $i j$ and $b c$ loops (Fig. 2C). In CCHFV, the HMIS conformation is further supported by a hydrogen bond network that involves the buried polar side chains of Asn ${ }^{1194}$ and Arg $^{1189}$ of the $c d$ loop in both the pre- and postfusion forms
(Fig. 2, A to C). The equivalent residues in MPRLV, Asn $^{769}$ and $\mathrm{Asn}^{764}$, recapitulate the same interactions in the postfusion form (26) but are solvent-exposed in the prefusion Gn-Gc heterodimer, where nonpolar side chains such as MPRLV Trp ${ }^{766}$, corresponding to CCHFV Trp ${ }^{1191}$, are instead buried (Fig. 2B). It is likely that Gn locks the domain II tip in the conformation shown in the MPRLV prefusion Gn-Gc complex, and that release of Gn results in the HMIS conformation seen in the pre- and postfusion forms of CCHFV. This suggests that the Gc monomer observed in the ternary complex corresponds to an activated prefusion form of CCHFV capable of insertion into the host membrane.

To experimentally test the role of residues suggested by the structure to be important for Gc function in membrane fusion, we established an assay to follow syncytia formation of cells expressing the CCHFV glycoproteins at their surface upon low-pH treatment. We used this assay to test single point mutations at the interface between domains I and III (at the turn of the postfusion hairpin) to explore their functional effect. Alanine substitutions of two conserved residues abrogated low-pHtriggered cell-cell fusion: $\mathrm{His}^{1479}$ on domain III, which makes a salt bridge with Glu ${ }^{1113}$ of domain I, and Trp ${ }^{1068}$ in the N-terminal tail, which projects into a pocket at the domain I/II boundary (Fig. 2, D to E). Gc-derived linear peptides spanning the N-terminal tail (amino acids 1041 to 1060 and 1061 to 1080) around the functionally important $\operatorname{Trp}^{1068}$ residue robustly react with $\mathrm{CCHFV}$-positive human sera (27), suggesting this site as a potential target for neutralizing antibodies. On the contrary, we saw no effect resulting from alanine substitution of $\mathrm{His}^{1398}$ at the binding pocket for the N-terminal tail and only a mild effect by alanine substitution of the glycosylation site $\operatorname{Asn}^{1563}$ on the stem (28) (Fig. 2, D to E).

We also tested the role of HMIS nonpolar side chains. Mutation to alanine of the highly conserved $\operatorname{Trp}^{1191}, \operatorname{Trp}^{1197}$, and $\operatorname{Trp}^{1199}$ exposed by the $c d$ loop, as well as Trp ${ }^{1365}$ and $\mathrm{Met}^{1362}$ exposed by the $i j$ loop (see Fig. 2C), strongly impaired low-pH-triggered syncytia formation relative to wild-type Gc when substituted individually (Fig. 2D). This result is in line with the functional effect of the corresponding 
residues of hantavirus Gc (Fig. 2B), which have been shown to be functionally required for target membrane insertion (22).

The residues exposed at the HMIS make up the epitope of $\mathrm{mAb} \mathrm{ADI}-37801$, which covers $627 \AA^{2}$ of surface area on Gc. Two-thirds of the epitope is buried by the three complementaritydetermining regions (CDRs) $\mathrm{H} 1, \mathrm{H} 2$, and $\mathrm{H} 3$ of the heavy chain, and the remainder by the light-chain CDRs L1 and L3 (Fig. 2F). There are four hydrogen bonds at the epitope-paratope interface (table S2). The core of the epitope is formed by the $c d$ loop, which contributes 10 amino acids, whereas the $b c$ loop contributes an additional two. The residues critical for membrane fusion-Trp ${ }^{1191}, \operatorname{Trp}^{1197}$, and $\operatorname{Trp}^{1199}$ of the $c d$ loop-are an integral part of the ADI37801 epitope (Fig. 2, A and F). Our structure
A
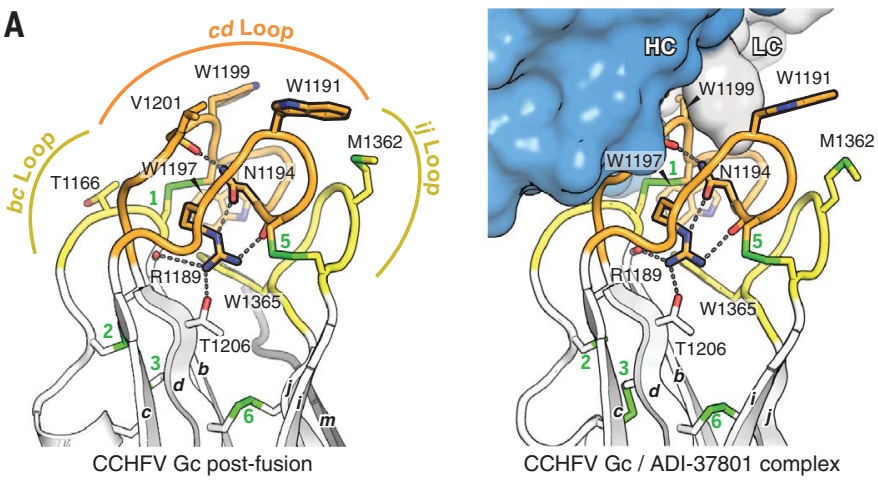

B

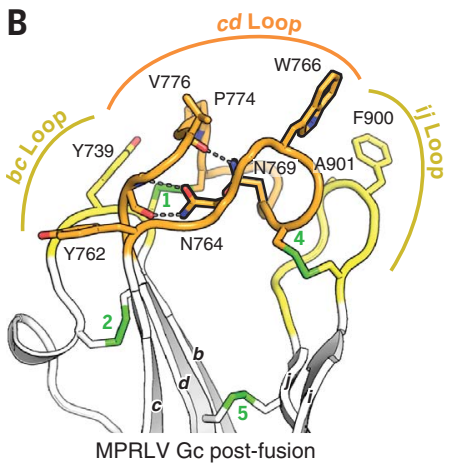

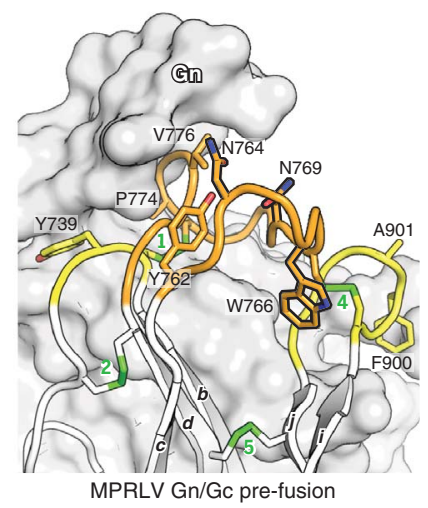

C

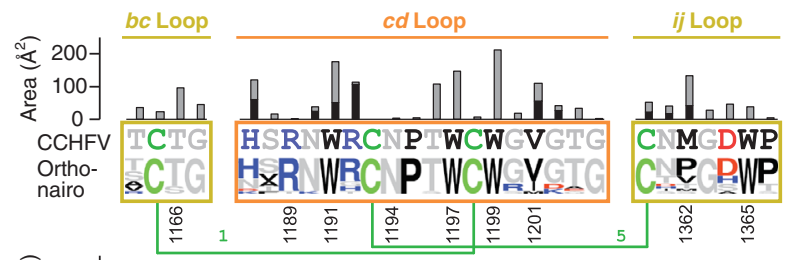

D

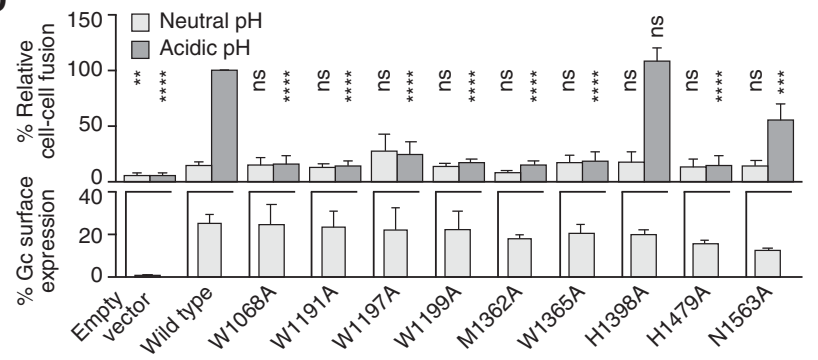

$\mathbf{E}$
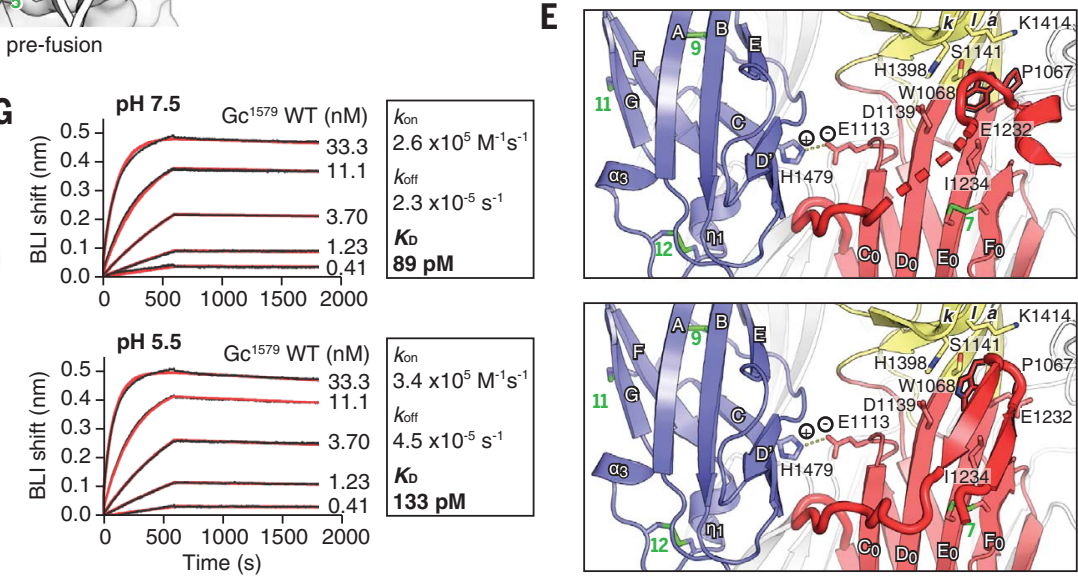

Fig. 2. ADI-37801 binds HMIS residues required for Gc-driven syncytia formation. (A) The CCHFV HMIS of the postfusion trimer (left) and in complex with ADI-37801 (right). In the left panel, W1191, W1197, and W1199, which were mutated to obtain the crystals, have been modeled for clarity. (B) Hantavirus fusion loops in the postfusion trimer forming the HMIS (left; PDB ID: 6y68, MPRLV structure) and in the prefusion Gn-Gc heterodimer, where the HMIS is not formed (right; PDB ID: 6y62) (15). (C) Fusion loop sequences of CCHFV Gc with consensus sequence logo for the Orthonairovirus (top) and Orthohantavirus (bottom) genera. The bar chart shows the exposed surface area per residue in pre- (hantavirus Gc) and postfusion (CCHFV and hantavirus Gc) structures. The accessible and buried surface per residue are represented in gray and black, respectively. Nonpolar residues, black; acidic, red; basic, blue; cysteines, green. (D) CCHFV Gc-induced syncytia formation by wild-type and indicated mutant Gc at neutral and acidic pH. The transfected cell surface expression is shown for each mutant below. (E) Details

of two alternative conformations of the $\mathrm{N}$-tail and a $\mathrm{pH}$-sensitive salt bridge between domains I and III. The helical conformation (top) is dominant, whereas the $\beta$-hairpin (bottom) is well defined in only two of the six polypeptide chains in the asymmetric unit of the monoclinic crystals obtained at $\mathrm{pH}$ 7.5. The view is the same as in Fig. 1C. (F) Interface between the ADI-37801 CDRs and the Gc fusion loops. The antibody heavy and light chain CDRs are colored blue and gray, respectively. CCHFV Gc is colored orange (cd loop) and yellow (bc loop). Polar interactions are denoted by dashed lines. (G) Biolayer interferometry (BLI) sensorgrams showing binding kinetics of CCHFV Gc ${ }^{1579}$ to ADI-37801 at pH 7.5 (top) or pH 5.5 (bottom). WT, wild-type; $K_{D}$, dissociation constant; $k_{\text {on }}$, on rate

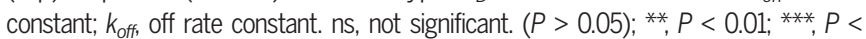
0.001; and ${ }^{* * *}, P<0.0001$ ). Single-letter abbreviations for the amino acid residues are as follows: A, Ala; C, Cys; D, Asp; E, Glu; F, Phe; G, Gly; H, His; I, lle; K, Lys; L, Leu; M, Met; N, Asn; P, Pro; Q, Gln; R, Arg; S, Ser; T, Thr; V, Val; W, Trp; and Y, Tyr. 
is thus consistent with yeast-display-based epitope mapping, which identified $\operatorname{Trp}^{1199}$ as critical for ADI-37801 binding (15).

Our ternary complex crystals grew at $\mathrm{pH}$ 5.6, suggesting that the complex of Gc and ADI37801 remains stable in the endosome during viral entry. Through biolayer interferometry (BLI) we confirmed that ADI-37801 binding is insensitive to mildly acidic conditions (Fig. $2 \mathrm{G}$ ). Taken together, the cell-cell fusion, structural, and kinetic data suggest that ADI-37801 inhibits endosomal membrane insertion of Gc by masking its fusion loops.

The x-ray structure showed that ADI-36121 binds laterally to the domain II base adjacent to the $\mathrm{Asn}^{1345}$ glycan and covers $943 \AA^{2}$ of surface area on Gc, 63 and $37 \%$ of which are buried by the heavy and light chains, respectively, involving all six CDRs (Fig. 3, A to B). The epitope is composed of 22 residues featuring 13 hydrogen bonds and one salt bridge at the interface (table S2). The structure is consistent with the yeast-display-based mutagenesis screen that identified Leu ${ }^{1307}$ and Ile ${ }^{1229}$ as important for ADI-36121 binding (Fig. 3B) (15).

Structural comparison shows that the ADI36121 epitope becomes entirely buried at the trimer interface upon formation of the postfusion trimer of Gc (Fig. 3, C to D). To experimentally confirm that the ADI-36121 epitope is inaccessible in the postfusion trimer, we used BLI to compare antibody binding with both monomeric and trimeric fractions of recombinant soluble Gc. The affinity of ADI36121 for the monomeric fraction was $\sim 200$ times higher than that for the trimeric fraction (Fig. 3E). The observed residual ADI-36121 binding to the trimeric fraction suggests contamination of the sample with Gc monomers, as trimeric and monomeric fractions eluted in partially overlapping peaks in sizeexclusion chromatography (fig. S2). Nevertheless, antibody binding likely outcompetes the trimerization process during viral infection, because the dissociation constant $\left(K_{\mathrm{D}}\right)$ of ADI-36121 for the Gc monomer is in the picomolar range at $\mathrm{pH} 7.5$ and 5.5 (Fig. 3F). These data suggest that ADI-36121 neutralizes CCHFV by blocking Gc homotrimerization in the endosome and preventing membrane fusion.

The CCHFV-neutralizing human antibodies described previously had been tentatively assigned to six different antigenic sites by using a homology model for Gc based on the MPRLV Gc structure (15). Our experimental structures confirm the proposed distribution of the epitopes among the three Gc domains and also reveal the neutralization mechanisms by showing that they map to the HMIS or other surfaces buried during Gc-driven membrane fusion (Fig. 4A). The dominant antigenic site 1 maps to the $c d$ loop (Fig. 4A), which is conserved across CCHFV strains as well as across members of the Orthonairovirus genus (Fig. 4, B
A

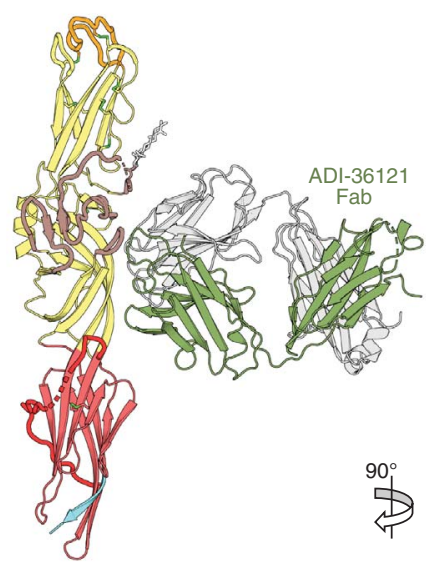

C

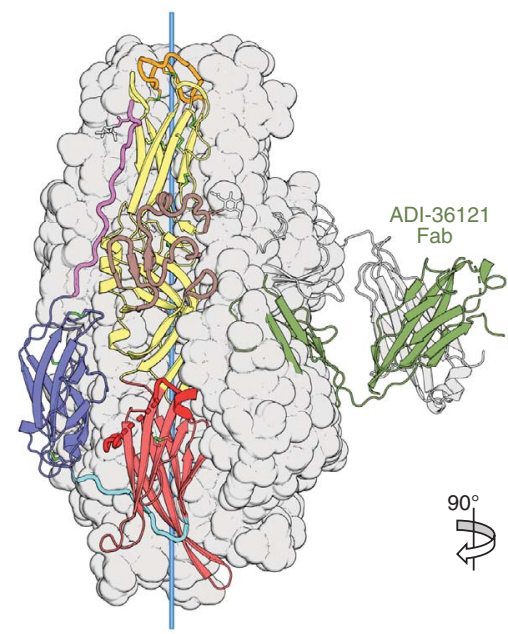

E
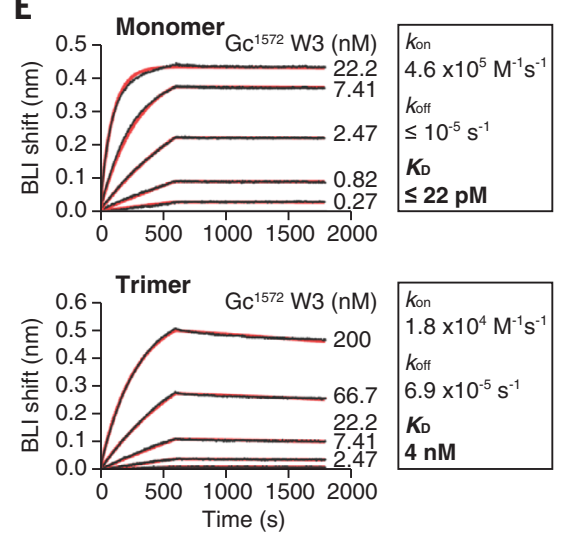

B

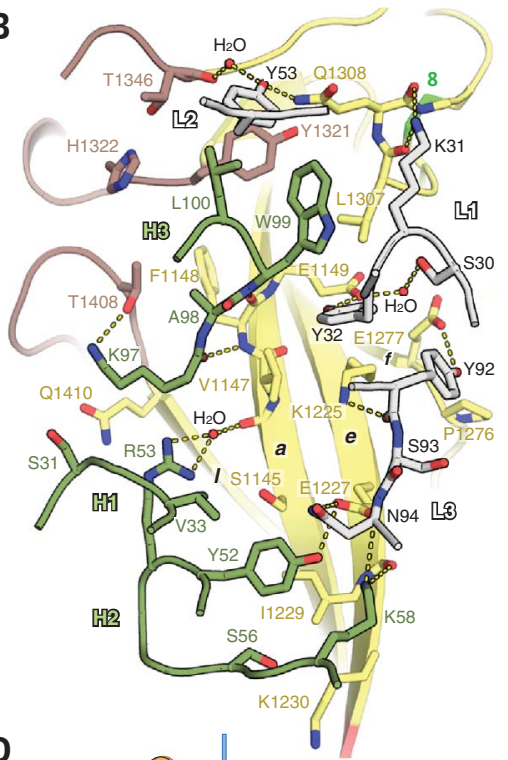

D

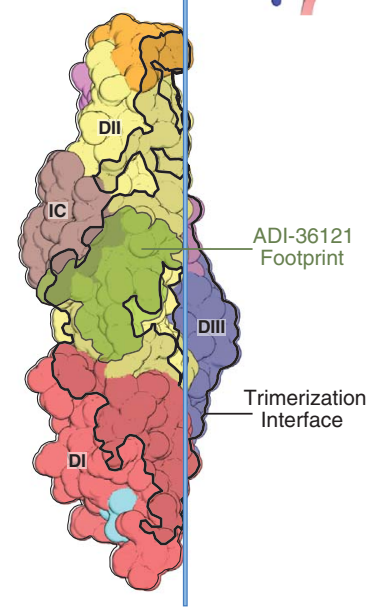

$\mathbf{F}$
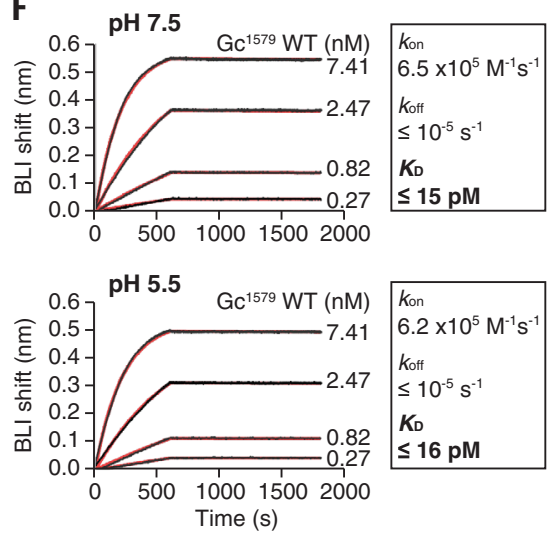

Fig. 3. The ADI-36121 epitope is buried at the trimer interface of the postfusion hairpin. (A) The CCHFV Gc monomer in a complex with the ADI-36121 Fab. (B) CDRs interacting with the Gc domain II base. Green and gray indicate heavy and light chains, respectively, and yellow indicates Gc domain II. Polar interactions are shown by dashes. (C) Superposition of the ADI-36121 complex with the Gc postfusion trimer. The trimer's front protomer is shown in ribbons colored according to domain, and the flanking protomers are shown as a white surface. (D) One protomer of the trimer shown as a surface colored according to domain, with the trimer interface outlined in black and the ADI-36121 footprint superposed in green, illustrating that the epitope is occluded in the trimer. (E) BLI sensograms showing binding kinetics of the monomeric fraction (top) or the trimeric fraction (bottom) of CCHFV Gc ${ }^{1572}$ W3 to ADI-36121 at pH 7.5. (F) BLI sensograms showing binding kinetics of CCHFV Gc $c^{1579}$ to ADI-36121 at pH 7.5 (top) or pH 5.5 (bottom). See materials and methods for details of the constructs used. 

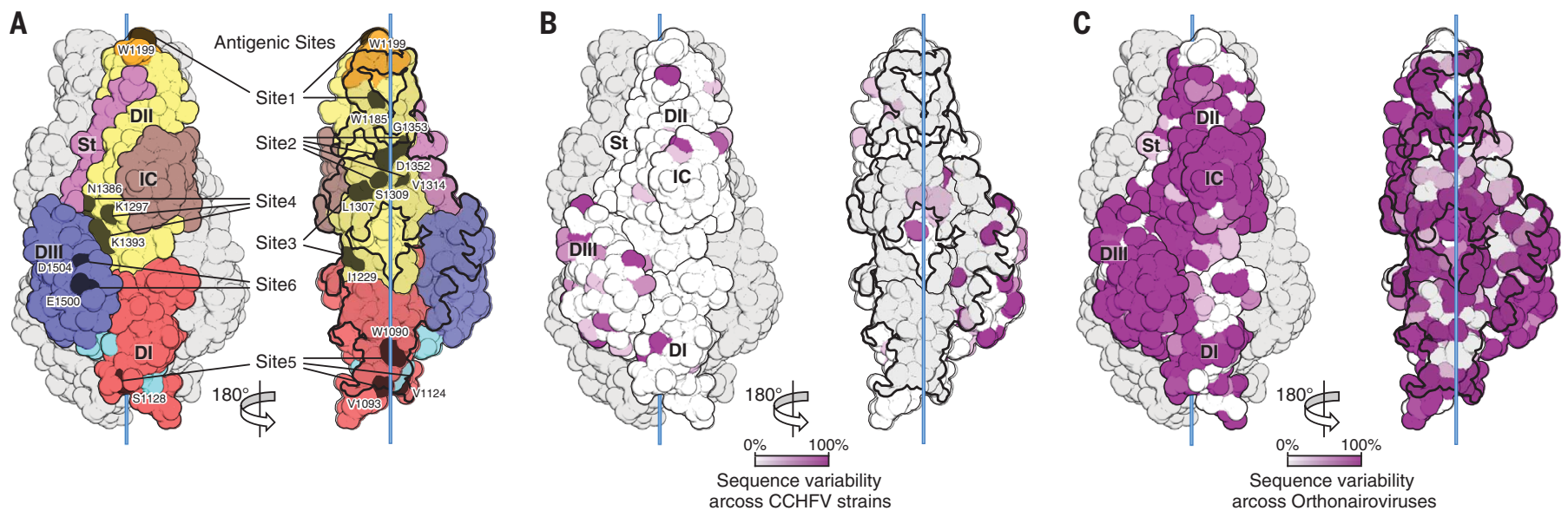

Fig. 4. The epitopes of CCHFV-neutralizing human antibodies map to Gc surfaces involved in driving membrane fusion. (A) Antigenic sites mapped on the surface of one CCHFV Gc protomer within the postfusion trimer. The trimer axis is shown in light blue. Only the front Gc subunit is shown in the right panel, after a $180^{\circ}$ rotation about the trimer axis. The trimer interface is outlined in black. St, Gc stem region. (B) Sequence variability across 15 representative CCHFV strains (fig. S3), color plotted on the Gc surface. (C) Sequence variability across 14 species in the Orthonairovirus genus (fig. S4). to C, and figs. S3 and S4). Antigenic sites 2 to 4 map to the domain II base, with sites 2 and 3 at the trimer core interface of postfusion Gc (Fig. 4A). The most potently neutralizing antibodies-including ADI-36121-target site 3 . Consistent with the high degree of conservation of its epitope across CCHFV strains (Fig. 4B), ADI-36121 displays highly potent crossclade neutralization (15), which makes it a viable candidate for clinical development. It remains to be investigated whether this $\mathrm{mAb}$ would be effective against nairoviruses from other serogroups, such as the veterinary pathogens Dugbe virus or Nairobi sheep disease virus, which can potentially spill over to humans (29), as Gc from these viruses carries several point mutations in the epitope (figs. S4 and S5). Site 4 maps to the opposite face of domain II, near the interface with domain III and the stem in the postfusion structure (Fig. 4A), suggesting that antibody binding would inhibit hairpin formation. Similar to sites 2 and 3, site 5 overlaps with the Gc trimer interface but lies within domain I (Fig. 4A). Moreover, antibody binding to site 5 likely restrains the conformational change of domain I during fusion (fig. S1A). Finally, site 6 maps to domain III, where antibody binding may sterically inhibit its translocation for postfusion hairpin formation (Fig. 4A). In addition to human antibodies, this site is likely also targeted by the broadly neutralizing murine antibody 11E7, which has been mapped to a Gc fragment encompassing both domain III and the stem (amino acids 1443 to 1566) (30). Because the epitope was sensitive to chemical reduction, it can now be assigned to the disulfide-stabilized domain III. Because domain III contains more sequence polymorphisms across CCHFV strains than the other Gc domains (Fig. 4B), cross-clade neutralization by site 6 antibodies may be more limited compared with the other sites. Although inhibition of binding to the currently unknown entry receptor for CCHFV may also play a role in neutralization, our findings are consistent with a neutralization mechanism that inhibits membrane fusion by blocking insertion of the HMIS into target membranes, by interfering with Gc trimerization, or by inhibiting postfusion hairpin formation.

Our structural data revealed that the HMIS of CCHFV Gc is at least transiently accessible on virus particles, as mAb ADI-37801 efficiently neutralizes the virus. However, the current paradigm is that the HMIS is protected from premature exposure by the companion protein Gn. The only available high-resolution structures of a bunyavirus Gn-Gc complex come from hantaviruses, and they indeed show that the conformation of the Gc domain II tip in interaction with Gn is such that the HMIS is not formed. Recent studies on Andes hantavirus have, however, shown a substantial degree of breathing, transiently exposing the HMIS at physiological temperatures (31). The strong structural similarity between their domain II tips (Fig. 2, A and C) suggests that comparable breathing dynamics can also be expected from CCHFV Gc. Because ADI-37801 neutralization was strain dependent (15) despite almost perfect conservation of the HMIS sequence across CCHFV strains (fig. S3), the breathing dynamics of the HMIS are likely controlled by sequences outside the fusion loops. Notably, strain-dependent breathing is also known to affect the neutralization potency of fusion loop antibodies in flaviviruses $(32,33)$.

Unlike the fusion loop antibody ADI-37801, the trimerization-inhibiting antibody ADI-36121 shows potent neutralization across CCHFV strains (15), indicating that accessibility of its epitope is not restricted by strain-dependent structural dynamics within the envelope. The ADI-36121 epitope on CCHFV Gc lies in the same position as the P-4G2 epitope on hantavirus Gc (fig. S6). Both antibodies bind to the same secondary structure elements on their respective Gc targets (fig. S6C), at a surface patch that is involved in lateral interspike contacts on the hantavirus glycoprotein lattice (fig. S6, A and D). This surface patch becomes buried in the Gc postfusion trimer in both cases. Low-resolution studies of Hazara virus, a nonpathogenic nairovirus, showed tetrameric spikes arranged in a square surface lattice (34), similar to that of hantaviruses. The hantavirus surface lattice was visualized at a higher resolution (26) and was very different from the icosahedral $\mathrm{T}=12 \mathrm{Gn}-\mathrm{Gc}$ lattice of the phlebovirus RVFV, for which relatively high-resolution structures are also available (35). Considering the similar square surface lattices of nairoviruses and hantaviruses and the structural similarity between the corresponding fusion proteins, it is reasonable to expect that comparable surfaces in CCHFV Gc are involved in lateral spikespike contacts (fig. S6, B and E). It is possible, therefore, that ADI-36121 perturbs the longrange order of the CCHFV envelope in a similar way as was shown for P-4G2 (36). Higherresolution cryo-electron tomography data on the nairovirus surface glycoprotein lattice are needed to identify the precise lateral spikespike contacts and confirm the predictions illustrated in fig. S6. Our study nevertheless raises notable parallels between these two zoonotic viruses despite their different lifestyles and reservoirs-one arthropod-borne and the other transmitted by small mammals-thus highlighting the power of comparative structural studies in understanding common features of emerging viruses. 
The combination of antibodies ADI-37801 and ADI-36121 displayed synergy in a neutralization assay (15). Moreover, a single dose of a bispecific antibody containing the variable domains of both ADI-36121 and ADI-37801 protected mice against $\mathrm{CCHFV}$ even when administered 24 hours after exposure, whereas the individual mAbs protected only in a prophylactic setting (15). To explain these findings, our structural analysis suggests that ADI-36121 binding indirectly influences the Gc fusion loop breathing dynamics by perturbation of the glycoprotein surface lattice in such a way that the HMIS becomes more exposed, allowing ADI-37801 to more easily recognize its epitope (fig. S6, E to F). Combination with ADI-36121 should therefore also broaden the reactivity of ADI-37801 with the various CCHFV strains, making these two antibodies strong candidates for therapeutic antibody cocktails. Describing CCHFV neutralization at the mechanistic level, our data guide the design of future therapeutic antibodies and will likewise support the design of protective $\mathrm{CCHFV}$ vaccines.

\section{REFERENCES AND NOTES}

1. O. Ergönül, Lancet Infect. Dis. 6, 203-214 (2006)

2. K. Tsergouli, T. Karampatakis, A. B. Haidich, S. Metallidis, A. Papa, J. Hosp. Infect. 105, 43-52 (2020)

3. E. Akinci, H. Bodur, H. Leblebicioglu, Vector Borne Zoonotic Dis. 13, 429-437 (2013)

4. D. A. Bente et al., Antiviral Res. 100, 159-189 (2013).

5. M. S. Mehand, F. Al-Shorbaji, P. Millett, B. Murgue, Antiviral Res. 159, 63-67 (2018).

6. E. Mancuso et al., Emerg. Infect. Dis. 25, 1418-1420 (2019),

7. A. Negredo et al., N. Engl. J. Med. 377, 154-161 (2017).

8. A. Negredo et al., Emerg. Infect. Dis. 25, 1177-1184 (2019)

9. P. Maes et al., Arch. Virol. 164, 927-941 (2019).

10. J. Ma et al., Nat. Med. 27, 434-439 (2021).

11. P. Guardado-Calvo, F. A. Rey, Adv. Virus Res. 98, 83-118 (2017).
12. R. J. G. Hulswit, G. C. Paesen, T. A. Bowden, X. Shi, Viruses 13 , $353(2021)$

13. M. J. Vincent et al., J. Virol. 77, 8640-8649 (2003).

14. A. Albornoz, A. B. Hoffmann, P.-Y. Lozach, N. D. Tischler, Viruses 8, 143 (2016).

15. J. M. Fels et al., Cell 184, 3486-3501.e21 (2021)

16. Y. Modis, Adv. Exp. Med. Biol. 790, 150-166 (2013)

17. P. Guardado-Calvo et al., Science 358, 663-667 (2017).

18. S. Halldorsson et al., Proc. Natl. Acad. Sci. U.S.A. 113, 7154-7159 (2016).

19. S. Bressanelli et al., EMBO J. 23, 728-738 (2004).

20. Y. Modis, S. Ogata, D. Clements, S. C. Harrison, Nature 427, 313-319 (2004)

21. D. L. Gibbons et al., Nature 427, 320-325 (2004).

22. P. Guardado-Calvo et al., PLOS Pathog. 12, e1005813 (2016)

23. S. Willensky et al., PLOS Pathog. 12, e1005948 (2016)

24. R. M. DuBois et al., Nature 493, 552-556 (2013).

25. M. Dessau, Y. Modis, Proc. Natl. Acad. Sci. U.S.A. 110 1696-1701 (2013).

26. A. Serris et al., Cell 183, 442-456.e16 (2020).

27. A. Fritzen et al., PLOS Negl. Trop. Dis. 12, e0006598 (2018)

28. B. R. Erickson, V. Deyde, A. J. Sanchez, M. J. Vincent, S. T. Nichol, Virology 361, 348-355 (2007).

29. N. J. MacLachlan, E. J. Dubovi, Eds., in Fenner's Veterinary Virology (Academic Press, ed. 5, 2017), pp. 41-424.

30. A. A. Ahmed et al., J. Gen. Virol. 86, 3327-3336 (2005).

31. E. A. Bignon, A. Albornoz, P. Guardado-Calvo, F. A. Rey, N. D. Tischler, elife 8, e46028 (2019).

32. K. A. Dowd, T. C. Pierson, Annu. Rev. Virol. 5, 185-207 (2018).

33. F. A. Rey, K. Stiasny, M. C. Vaney, M. Dellarole, F. X. Heinz, EMBO Rep. 19, 206-224 (2018)

34. E. K. Punch et al., J. Biol. Chem. 293, 9937-9944 (2018).

35. S. Halldorsson et al., Nat. Commun. 9, 349 (2018).

36. I. Rissanen et al., elife 9, e58242 (2020)

\section{ACKNOWLEDGMENTS}

We thank members of the McLellan and Rey laboratories for providing helpful comments on the manuscript; F. Agou from the Chemogenomic and Biological Screening platform at Institut

Pasteur; the staff of the Crystallography platform at Institut Pasteur; the synchrotron beamlines PX2 at SOLEIL (St. Aubin, France), ID23-1 at the ESRF (Grenoble, France), and 19-ID at Argonne operated by UChicago Argonne, LLC for the US Department of Energy (DOE), Office of Biological and Environmental Research under contract DE-AC02-06CH11357.

Funding: This work was supported by National Institutes of Health award U19 Al142777 to J.S.M., Z.A.B., K.C., and L.M.W., as well as by Institut Pasteur, CNRS and grant ANR-10-LABX-62-10 IBEID to
F.A.R and by the LabEx Ecofect (ANR-11-LABX-0048) of the "Université de Lyon," within the program "Investissements d'Avenir" (ANR-11-IDEX-0007) operated by the French National Research Agency (ANR), to F.-L.C. Research was funded in part by Welch Foundation grant F-0003-19620604 awarded to J.S.M. The Pasteur-Cantarini 24-month fellowship was granted to J.H., who was further supported by the Région lle de France (Domaine d'intérêt majeur - innovative technologies for life sciences, DIM 1HEALTH). Author contributions: Conceptualization: A.K.M., J.H., J.S.M., and F.A.R.; Methodology and Formal Analysis: A.K.M., J.H., N.F., P.G.-C., F.-L.C., J.S.M., and F.A.R.; Investigation: A.K.M., J.H., N.F., P.G.-C., and A.H.; Resources: J.M.F., D.P.M., and D.M.A. Writing: A.K.M., J.H., J.S.M., and F.A.R.; Reviewing and Editing: all authors: Visualization J.H., A.K.M., J.S.M., and F.A.R.;

Supervision: F.A.R., J.S.M., F.- L.C., K.C., L.M.W., Z.A.B., and P.G.-C.: Funding Acquisition: F.A.R., J.S.M., F.-L.C., K.C., L.M.W., Z.A.B., and J.H. Competing interests: F.A.R. is a board member and shareholder of EureKARE and MELETIUS Therapeutics. D.M.A. and Z.A.B. are employees and shareholders at Mapp Biopharmaceutical, Inc. L.M.W. is an employee at Adimab, LLC: D.P.M. and L.M.W. are shareholders of Adimab, LLC. K.C. has consulted for Axon Advisors, is a member of the scientific advisory boards of Integrum Scientific LLC, and Biovaxys Technology Corp., LLC; K.C., and J.S.M. are members of the scientific advisory board of the Pandemic Security Initiative of Celdara, LLC. A.K.M., J.M.F., D.P.M., D.M.A., Z.A.B.

L.M.W., K.C., and J.S.M. are listed as inventors on a pending patent application with provisional number 63/021,004, entitled "AntiCrimean-Congo Hemorrhagic Fever Virus antibodies, and methods of their generation and use". Data and materials availability: Atomic coordinates of the reported structures have been deposited in the Protein Data Bank under accession codes 7A59, 7A5A, 7L7R, and 7KX4. Antibodies ADI-37801 and ADI-36121 are available from the corresponding author J.S.M. under a material transfer agreement with the University of Texas at Austin.

\section{SUPPLEMENTARY MATERIALS}

science.org/doi/10.1126/science.abl6502

Materials and Methods

Figs. S1 to S6

Tables S1 and S2

References (37-57)

MDAR Reproducibility Checklist

View/request a protocol for this paper from Bio-protocol.

27 July 2021; accepted 8 November 2021

Published online 18 November 2021

10.1126/science.abl6502 


\title{
Science
}

\section{Structural basis of synergistic neutralization of Crimean-Congo hemorrhagic fever virus by human antibodies}

Akaash K. MishraJan HellertNatalia FreitasPablo Guardado-CalvoAhmed HaouzJ. Maximilian FelsDaniel P. MaurerDafna M. AbelsonZachary A. BornholdtLaura M. WalkerKartik ChandranFrançois-Loïc CossetJason S. McLellanFelix A. Rey

Science, 375 (6576), • DOI: 10.1126/science.abl6502

\begin{abstract}
A block to viral cell entry
Crimean-Congo hemorrhagic fever virus is a tickborne virus that can cause severe disease and even death in humans. Disease occurrence is linked to the geographic range of the tick vector, and climate change may increase this range. Infection of host cells requires the fusion glycoprotein Gc, which is the main target of neutralizing antibodies. Mishra et al. build on previous work that identified a combination of two Gc-targeting antibodies that gave postexposure protection in an animal model. The authors determined the structure of the antigen-binding fragments of the two antibodies bound to a prefusion form of $\mathrm{Gc}$ and also the structure of $\mathrm{Gc}$ after the conformational change into the trimeric postfusion form. The structures show how the antibodies work together to block membrane fusion. - VV
\end{abstract}

View the article online

https://www.science.org/doi/10.1126/science.abl6502 Permissions

https://www.science.org/help/reprints-and-permissions 